\title{
Fatty liver disease in severe obese patients: Diagnostic value of abdominal ultrasound
}

\author{
Alessandro de Moura Almeida, Helma Pinchemel Cotrim, Daniel Batista Valente Barbosa, \\ Luciana Gordilho Matteoni de Athayde, Adimeia Souza Santos, Almir Galvão Vieira Bitencourt, \\ Luiz Antonio Rodrigues de Freitas, Adriano Rios, Erivaldo Alves
}

\begin{abstract}
Alessandro de Moura Almeida, Helma Pinchemel Cotrim, Daniel Batista Valente Barbosa, Luciana Gordilho Matteoni de Athayde, Adimeia Souza Santos, Almir Galvão Vieira Bitencourt, Luiz Antonio Rodrigues de Freitas, Faculdade de Medicina da Bahia, Universidade Federal da Bahia, Salvador, Bahia 41830-020, Brasil

Luiz Antonio Rodrigues de Freitas, Centro de Pesquisa Gonçalo Muniz-Fundação Osvaldo Cruz, Salvador, Bahia 41830-020, Brasil

Adriano Rios, Erivaldo Alves, Núcleo de Cirurgia da Obesidade, Salvador, Bahia 41830-020, Brasil

Author contributions: Almeida AM and Cotrim HP designed research and wrote the paper; Almeida AM, Barbosa DBV, Athayde LGM, Santos AS and Bitencourt AGV performed research; Freitas LAR analyzed all histological samples; Rios A and Alves E performed the preoperative evaluation of patients and the surgeries. Supported by PAPES/CNPq n ${ }^{\circ}$ 400267/2006-3

Correspondence to: Helma Pinchemel Cotrim, PhD, Faculdade de Medicina da Bahia, Universidade Federal da Bahia, Avenida Tancredo Neves, Ed. Salvador Trade Center, Torre Norte-Sala 717, Salvador, Bahia 41830-020, Brazil. helmacotrim@terra.com.br Telephone: +55-71-32762373 Fax: +55-71-32762373

Received: August 7, 2007

Revised: January 18, 2008
\end{abstract}

US was associated to advanced grades of steatosis on histology $(P=0.016)$.

CONCLUSION: Preoperative abdominal US in our series has not shown to be an accurate method for the diagnosis of hepatic steatosis in severe obese patients. Until another non-invasive method demonstrates better sensitivity and specificity values, histological evaluation may be recommended to these patients undergoing bariatric surgery.

\section{(c) 2008 WJG. All rights reserved.}

Key words: Bariatric surgery; Obesity; Hepatic steatosis; Abdominal ultrasound diagnosis; Fatty liver

Peer reviewer: Eddie Wisse, Professor, Irisweg 16, Keerbergen 3140, Belgium

Almeida AM, Cotrim HP, Barbosa DBV, Athayde LGM, Santos AS, Bitencourt AGV, Freitas LAR, Rios A, Alves E. Fatty liver disease in severe obese patients: Diagnostic value of abdominal ultrasound. World J Gastroenterol 2008; 14(9): 1415-1418 Available from: URL: http://www.wjgnet.com/1007-9327/14/1415.asp DOI: http:// dx.doi.org/10.3748/wjg.14.1415

\section{INTRODUCTION}

Non-alcoholic fatty liver disease (NAFLD) has been recognized as an important and common clinical entity, affecting approximately $20 \%$ of the general population ${ }^{[1]}$. The prevalence of NAFLD in obese people has been estimated in 60\%-95\% and, currently, NAFLD has been suggested to be the liver component of the metabolic syndrome ${ }^{[2,3]}$. It has a large spectrum, ranging from simple hepatic steatosis to steatohepatitis (NASH) and cirrhosis.

Liver biopsy and histological evaluation have been considered the better methods for the diagnosis of steatosis and to establish the prognosis of NAFLD. However, there are controversies about the indication of biopsy in clinical practice, due the lack of an effective medical therapy for NAFLD and the risks associated with this procedure ${ }^{[4]}$.

Nevertheless, various imaging modalities have been used to diagnose the presence of fat in the liver, as ultrasonography, computerized tomography scan and magnetic resonance imaging. Abdominal ultrasound (US), the 
cheapest method, has been the most common modality used in clinical practice. Some parameters allow the diagnosis of fatty liver disease with a sensibility of $83 \%$ and a specificity of $100 \%$ : a diffuse hyperechoic echotexture; deep attenuation; increased liver echotexture compared with the kidney; and vascular blurring ${ }^{[5]}$. However, lower sensitivity values of US have been demonstrated among severe obese people ${ }^{[6]}$. The present study aimed to evaluate the reliability of abdominal US when it is compared to histology to diagnose hepatic steatosis in these patients and its relation to histological grades of steatosis.

\section{MATERIALS AND METHODS}

\section{Study group selection}

A consecutive series of obese patients who underwent bariatric surgery from October 2004 to May 2005 was selected. The eligible criteria for inclusion were: age above 18 years, preoperative abdominal US, and liver biopsy during the surgery and signer agreement to participate the study. All patients had body mass index above $40 \mathrm{~kg} / \mathrm{m}^{2}$, or $35 \mathrm{~kg} / \mathrm{m}^{2}$ associated to others conditions (hypertension, diabetes, dyslipidemia or sleep apnea $)^{[7]}$. Patients with alcohol intake above $20 \mathrm{~g} / \mathrm{d}$ or those who had other chronic liver diseases (HBV or HCV infection, hemochromatosis, autoimmune hepatitis, Wilson's disease, primary biliary cirrhosis, $\alpha-1$ antitrypsin-deficiency) were excluded. The same surgeon team made all surgeries. Abdominal US was performed in all patients as part of routine preoperative time and different radiologists carried them out.

This study was performed in accordance with a protocol approved by Ethics Committee for Medical Research of Gonçalo Muniz Research Center. All included patients have consented to their participation in this study.

\section{Clinical evaluation}

The parameters studied included: age, gender, height, weight, waist circumference, history of hypertension, dyslipidemia, diabetes and drugs use. Laboratory evaluation included: hemoglobin, hematocrite, leucocytes, prothrombin time, ASL, ALT, total bilirubin, albumin, total cholesterol, HDL-cholesterol, triglycerides, fasting plasma glucose and insulin. Abdominal US exam was performed in all patients. Insulin resistance was calculated using Homeostasis Model Assessment Index (HOMA-IR). Patients were categorized as insulin resistant if the HOMA-IR value was equal or greater than 3.0, as previously described ${ }^{[8]}$. Ultrasonographic definition of steatosis was based on diagnosis criteria usually used in clinical practice, as mentioned above ${ }^{[5]}$.

\section{Liver biopsy and histological analysis}

An intraoperative wedge biopsy was obtained at the beginning of the surgery and all samples were processed and examined by a single pathologist, using hematoxylin-eosin stain. Hepatic steatosis in the biopsy specimens, if present, was graded according the number of involved hepatocytes: Grade I (steatosis in 5\%-25\% of hepatocytes); Grade II (steatosis in 25\%-50\% of hepatocytes); Grade III (steatosis in $50 \%-75 \%$ of hepatocytes); Grade IV (steatosis in more than $75 \%$ of hepatocytes).

\section{Statistical analysis}

Data were processed and analyzed using the Statistical Package for Social Science program, version 9.0 (SPSS Inc. Chicago, Illinois, USA). Descriptive statistics of the included variables has been carried out. The US and histological findings of steatosis were compared, considering histology as the gold standard. Subsequently, the sensibility, specificity, positive predictive value and negative predictive value for the US in the diagnosis of hepatic steatosis and theirs 95\% confidence intervals were calculated using EPI INFO v6.0 (CDC, USA). A Chi-square test was used to compare categorical variables. All statistical methods were two-tailed and the statistical significance was obtained when $P<0.05$.

\section{RESULTS}

Among the 123 severe obese patients with histological and abdominal US evaluation, 105 were finally included. Seventeen patients were excluded because they had a history of alcohol intake above $20 \mathrm{~g} / \mathrm{d}$, and one by hepatitis B infection (HBsAg positive).

Demographic and clinical profiles of these 105 individuals are shown in Table 1 . The US examination was normal in $38(36.2 \%)$ cases. Steatosis on US was described in 62 $(59.0 \%)$ cases. Others imaging findings were: hepatomegaly in $8(7.6 \%)$; colelithiasis in $14(13.3 \%)$; and renal cyst in 3 $(2.9 \%)$ cases.

The histological prevalence of steatosis was $89.5 \%$. The sensitivity and specificity of abdominal US for the diagnosis of hepatic steatosis were, respectively, 64.9\% (95\% CI: 54.9-74.3) and 90.9\% (95\% CI: 57.1-99.5). The positive and negative predictive values were, respectively, 98.4\% (95\% CI: 90.2-99.9) and 23.3\% (95\% CI: 12.3-39.0). A false positive rate was found in 9.1\% (95\% CI: 0.5-37.3) and a false negative rate in $35.1 \%$ (95\% CI: 26.0-45.2).

Table 2 shows the influence of body mass index on accuracy of abdominal US in the diagnosis of hepatic steatosis. The prevalence of steatosis in patients with body mass index between $35.0 \mathrm{~kg} / \mathrm{m}^{2}$ and $39.9 \mathrm{~kg} / \mathrm{m}^{2}$ and in patients with body mass index above $40 \mathrm{~kg} / \mathrm{m}^{2}$ was $83.3 \%$ and $91.3 \%$, respectively.

All individuals were separated into two groups, according to the median of waist circumference (below and above the median value) and the sensibility, specificity, positive predictive value and negative value were analyzed in each group. These results are demonstrated in Table 3. The prevalence of steatosis in patients below and above the median value for waist circumference was $81.1 \%$ and $94.6 \%$, respectively.

The presence of steatosis on US was associated with advanced grades of steatosis in the biopsy specimens $(P=0.016)$, as shown in Table 4 .

\section{DISCUSSION}

Abdominal US has been largely used in clinical practice and in protocols of investigation of patients with NAFLD because it is a cheap and a safe method. As a screening test, its major requirement is a high degree of sensitivity and specificity. In non-obese patients the values of sensitivity 
Table 1 Demographic and clinical characteristics of severe obese patients who underwent to bariatric surgery

\begin{tabular}{lc} 
Characteristics & Value \\
\hline Female gender - $n(\%)$ & $79(75.2)$ \\
Age, in yr - mean \pm SD & $37.2 \pm 10.6$ \\
BMI, in $\mathrm{kg} / \mathrm{m}^{2}$ - mean \pm SD & $43.8 \pm 5.2$ \\
Elevated waist circumference - $n(\%)$ & $105(100)$ \\
Hypertension - $n(\%)$ & $55(52.4)$ \\
Diabetes - $n(\%)$ & $10(9.5)$ \\
Dyslipidemia - $n(\%)$ & $75(71.4)$ \\
Exposure to chemicals - $n(\%)$ & $10(9.5)$ \\
Elevated transaminases - $n(\%)$ & $30(28.6)$ \\
HDL cholesterol - mean \pm SD & $46.5 \pm 5.2$ \\
Triglyceride - mean \pm SD & $157.3 \pm 82.8$ \\
Fasting plasma glucose level - mean \pm SD & $102.6 \pm 40.8$ \\
Insulin resistance - $n(\%)$ & $52(49.5)$ \\
\hline
\end{tabular}

BMI: Body mass index.

Table 2 Levels of sensibility, specificity, PPV and NPV for the ultrasound in the diagnosis of hepatic steatosis by body mass index (BMI) values

\begin{tabular}{|c|c|c|c|c|}
\hline \multirow[t]{2}{*}{ Variables } & \multicolumn{2}{|c|}{$35.0 \mathrm{~kg} / \mathrm{m}^{2} \leqslant \mathrm{BMI} \leqslant 40 \mathrm{~kg} / \mathrm{m}^{2}$} & \multicolumn{2}{|c|}{$\mathrm{BMI} \geqslant 40 \mathrm{~kg} / \mathrm{m}^{2}$} \\
\hline & Value (\%) & $95 \% \mathrm{CI}$ & Value (\%) & $95 \% \mathrm{CI}$ \\
\hline Sensibility & 65 & $40.9-83.7$ & 64.4 & $52.2-75.0$ \\
\hline Specificity & 75 & 21.9-98.7 & 100 & $56.1-100$ \\
\hline PPV & 92.9 & 64.2-99.6 & 100 & $90.6-100$ \\
\hline NPV & 30 & $8.1-64.6$ & 21.2 & $9.6-39.4$ \\
\hline
\end{tabular}

PPV: Positive predictive value; NPV: Negative predictive value; 95\% CI: Confidence interval of $95 \%$.

and specificity of US range from $83 \%$ to $94 \%$, and $84 \%$ to $100 \%$ respectively ${ }^{[5,9]}$.

The present study with severely obese patients evaluated the sensitivity and specificity of abdominal US for the diagnosis of hepatic steatosis and its relation to histology. The results showed a low performance of US to diagnosis steatosis, however similar results have been demonstrated in patients with body mass index ranging from $35.0 \mathrm{~kg} / \mathrm{m}^{2}$ to $82.2 \mathrm{~kg} / \mathrm{m}^{2}$, where the frequency of steatosis was $91.4 \%{ }^{[6]}$. In this case, sensitivity and specificity of US in the diagnosis of steatosis was $49.1 \%$ and $75 \%$, respectively ${ }^{[6]}$. These values are also demonstrated (sensitivity: $43 \%$; specificity: $79 \%$ ) for the diagnosis hepatic steatosis in patients infected with hepatitis $C$ virus ${ }^{[10]}$.

Several hypotheses may explain this low performance of US in severe obese people. The diagnosis made by different radiologists may introduce variability in interpretation of images. This could be related to the experience of each radiologist and to the lack of clear standards for the diagnosis of hepatic steatosis ${ }^{[11]}$. The second hypothesis is related to the adipose tissue thickness that may cause technical difficulties for the performance of this exam. The thick layers of subcutaneous fat in obese people may mislead the examiner's judgment of liver echogenicity, as cited in visualizing of the abdominal aorta $^{[12]}$ and renal carcinoma ${ }^{[13]}$. The image quality rate also has been discussed and different results have as been found. The analysis of 140 patients, who underwent
Table 3 Levels of sensibility, specificity, PPV and NPV for the ultrasound in the diagnosis of hepatic steatosis by waist circumference (WC) in severe obese patients

\begin{tabular}{lcccccc}
\hline \multirow{2}{*}{ Variables } & \multicolumn{2}{c}{$\mathbf{W C} \leqslant \mathbf{1 1 6 . 9} \mathbf{~ k g / \mathbf { m } ^ { \mathbf { 2 } }}$} & & \multicolumn{2}{c}{$\mathbf{W C} \geqslant \mathbf{1 1 7 . 0} \mathbf{~ k g} / \mathbf{m}^{\mathbf{2}}$} \\
\cline { 2 - 3 } \cline { 6 - 7 } & Value (\%) & $\mathbf{9 5 \%} \mathbf{C I}$ & & Value (\%) & $\mathbf{9 5 \%} \mathbf{C I}$ \\
\hline Sensibility & 56.7 & $37.7-74.0$ & & 80 & $62.5-90.9$ \\
Specificity & 85.7 & $42.0-99.2$ & & 100 & $19.8-100$ \\
PPV & 94.4 & $70.6-99.7$ & & 100 & $85.0-100$ \\
NPV & 31.6 & $13.6-56.5$ & & 22.2 & $3.9-59.8$ \\
\hline
\end{tabular}

PPV: Positive predictive value; NPV: Negative predictive value; 95\% CI: Confidence interval of $95 \%$.

Table 4 Ultrasound evaluation and grades of steatosis among severe obese patients with steatosis on biopsy (\%)

\begin{tabular}{lcc}
\hline \multirow{2}{*}{ Imaging profile } & Grade of steatosis on histological evaluation \\
\cline { 2 - 3 } & Grade I / II & Grade III/IV \\
\hline Ultrasound without steatosis & 97.00 & 3.00 \\
Ultrasound with steatosis & 77.00 & 23.00 \\
\hline
\end{tabular}

$P=0.016$.

abdominal US, showed that obesity was also associated with a poor sonographic image $\mathrm{e}^{[14]}$. However, another study did not find the same results ${ }^{[15]}$. Finally, the majority of screening values described in literature were calculated in patients with suspected liver disease. It is more appropriate to use groups of patients resembling those that have been investigated in clinical practice.

This investigation found a higher accuracy of US for the diagnosis of hepatic steatosis in patients with central obesity or more elevated waist circumference. This could be explained by the association between steatosis on ultrasonographic evaluation and histological evidence of steatosis in those patients, as previously showed ${ }^{[0,16]}$. In a multivariate model involving patients with hepatitis $C$, Hepburn found that the only statistically significant factor associated with steatosis on US was histological grade, with an odds-ratio of $3.6^{[10]}$. In addition, a better performance of ultrasound associated to a more elevated prevalence of NAFLD was also described in obese people undergoing bariatric surgery ${ }^{[6]}$, obtaining results as high as those found in non-obese people ${ }^{[5,9]}$.

The prevalence of steatosis among the patients in this study was elevated $(89.5 \%)$ and the central obesity was frequent, as previously described studies ${ }^{[6,17,18]}$. Moreover, the prevalence of NAFLD also has been correlated to body mass index. These results are relevant when we considered that obesity is associated to increased visceral adiposity, free fatty acids and hyperinsulinemia, which are involved in the pathogenesis of NAFLD ${ }^{[19,20]}$.

In conclusion, the results suggest that abdominal US may not be considered an accurate method for the diagnosis of hepatic steatosis in severe obese patients. The liver biopsy and histological evaluation should be recommended to these patients undergoing bariatric surgery, until other non-invasive method demonstrates better sensitivity and specificity values. 


\section{COMMENTS}

\section{Background}

Non-alcoholic fatty liver disease (NAFLD) is a common entity among severe obese patients. Although liver biopsy was the best method for its diagnosis, various imaging modalities have been used to diagnose the presence of fat in the liver, and abdominal ultrasound (US) is the most used in clinical practice. Thus, it is important to elucidate if the abdominal US is a good method to diagnose hepatic steatosis in these patients.

\section{Research frontiers}

This study reported the findings of 105 patients with a histological prevalence of steatosis of $89.5 \%$. Low sensitivity and negative predictive rates of US in the diagnosis of hepatic steatosis were described and a better performance of US was associated to advanced grades of steatosis on histology. More accurate methods may change this data.

\section{Innovations and breakthroughs}

Although the reliability of abdominal US for diagnose hepatic steatosis was previously reported, the current study showed different values of specificity and sensibility on severe obese patients by body mass index and waist circumference status and the relationship between grade of steatosis on histology and its presence on US evaluation.

\section{Applications}

Abdominal US results should be carefully analysed in obese patients. Because of the high prevalence of non-alcoholic fatty liver disease, a more accurate method for its diagnosis, as liver biopsy, is recommended in patients undergoing bariatric surgery.

\section{Terminology}

NAFLD means non-alcoholic fatty liver disease; NASH is named as non-alcoholic steatohepatits; HOMA-IR is homeostasis model assessment index - insulin resistance.

\section{Peer review}

The authors evaluate the use of US in severely obese patients in order to diagnose hepatic steatosis. They compare histological findings, which they consider to be the golden standard, with US data of the same patients. They observe 123 cases and conclude that US may not be considered an accurate method for the diagnosis of steatosis in these patients. The goals of the study, the materials and methods, results and other parts of the manuscript are well formulated and explain the study in well chosen terms. As a histologist, it is nice to hear that histology is the method of choice for this diagnosis, in spite of the fact that US based diagnosis would have been a very direct, non-invasive and cheap method to use.

\section{REFERENCES}

1 Bedogni G, Miglioli L, Masutti F, Tiribelli C, Marchesini G, Bellentani S. Prevalence of and risk factors for nonalcoholic fatty liver disease: the Dionysos nutrition and liver study. Hepatology 2005; 42: 44-52

2 Hornboll P, Olsen TS. Fatty changes in the liver: the relation to age, overweight and diabetes mellitus. Acta Pathol Microbiol Immunol Scand 1982; 90: 199-205

3 Marchesini G, Brizi M, Morselli-Labate AM, Bianchi G, Bugianesi E, McCullough AJ, Forlani G, Melchionda N. Association of nonalcoholic fatty liver disease with insulin resistance. Am J Med 1999; 107: 450-455

4 Sanyal AJ. AGA technical review on nonalcoholic fatty liver disease. Gastroenterology 2002; 123: 1705-1725

5 Yajima Y, Ohta K, Narui T, Abe R, Suzuki H, Ohtsuki M. Ultrasonographical diagnosis of fatty liver: significance of the liver-kidney contrast. Tohoku J Exp Med 1983; 139: 43-50

6 Mottin CC, Moretto M, Padoin AV, Swarowsky AM, Toneto MG, Glock L, Repetto G. The role of ultrasound in the diagnosis of hepatic steatosis in morbidly obese patients. Obes Surg 2004; 14: 635-637

7 Hubbard VS, Hall WH. Gastrointestinal Surgery for Severe Obesity. Obes Surg 1991; 1: 257-265

8 Guidorizzi de Siqueira AC, Cotrim HP, Rocha R, Carvalho FM, de Freitas LA, Barreto D, Gouveia L, Landeiro L. Nonalcoholic fatty liver disease and insulin resistance: importance of risk factors and histological spectrum. Eur J Gastroenterol Hepatol 2005; 17: 837-841

9 Saverymuttu SH, Joseph AE, Maxwell JD. Ultrasound scanning in the detection of hepatic fibrosis and steatosis. $\mathrm{Br}$ Med J (Clin Res Ed) 1986; 292: 13-15

10 Hepburn MJ, Vos JA, Fillman EP, Lawitz EJ. The accuracy of the report of hepatic steatosis on ultrasonography in patients infected with hepatitis $C$ in a clinical setting: a retrospective observational study. BMC Gastroenterol 2005; 5: 14

11 Lupsor M, Badea R. Imaging diagnosis and quantification of hepatic steatosis: is it an accepted alternative to needle biopsy? Rom J Gastroenterol 2005; 14: 419-425

12 Paslawski M, Krzyzanowski K, Kesik J, Zlomaniec J. Limitations in ultrasonographic evaluation of the abdominal aortic aneurysms. Ann Univ Mariae Curie Sklodowska 2004; 59 42-47

13 Webb JA, Murray A, Bary PR, Hendry WF. The accuracy and limitations of ultrasound in the assessment of venous extension in renal carcinoma. Br J Urol 1987; 60: 14-17

14 Shmulewitz A, Teefey SA, Robinson BS. Factors affecting image quality and diagnostic efficacy in abdominal sonography: a prospective study of 140 patients. J Clin Ultrasound 1993; 21: 623-630

15 Hann LE, Bach AM, Cramer LD, Siegel D, Yoo HH, Garcia R. Hepatic sonography: comparison of tissue harmonic and standard sonography techniques. AJR Am J Roentgenol 1999; 173: 201-206

16 Joseph AE, Saverymuttu SH, al-Sam S, Cook MG, Maxwell JD. Comparison of liver histology with ultrasonography in assessing diffuse parenchymal liver disease. Clin Radiol 1991; 43: $26-31$

17 Boza C, Riquelme A, Ibanez L, Duarte I, Norero E, Viviani P, Soza A, Fernandez JI, Raddatz A, Guzman S, Arrese M. Predictors of nonalcoholic steatohepatitis (NASH) in obese patients undergoing gastric bypass. Obes Surg 2005; 15: 1148-1153

18 Ong JP, Elariny H, Collantes R, Younoszai A, Chandhoke V, Reines HD, Goodman Z, Younossi ZM. Predictors of nonalcoholic steatohepatitis and advanced fibrosis in morbidly obese patients. Obes Surg 2005; 15: 310-315

19 Youssef WI, McCullough AJ. Steatohepatitis in obese individuals. Best Pract Res Clin Gastroenterol 2002; 16: 733-747

20 Frantzides CT, Carlson MA, Moore RE, Zografakis JG, Madan AK, Puumala S, Keshavarzian A. Effect of body mass index on nonalcoholic fatty liver disease in patients undergoing minimally invasive bariatric surgery. J Gastrointest Surg 2004; 8: 849-855

S- Editor Li DL L- Editor Alpini GD E- Editor Lu W 